
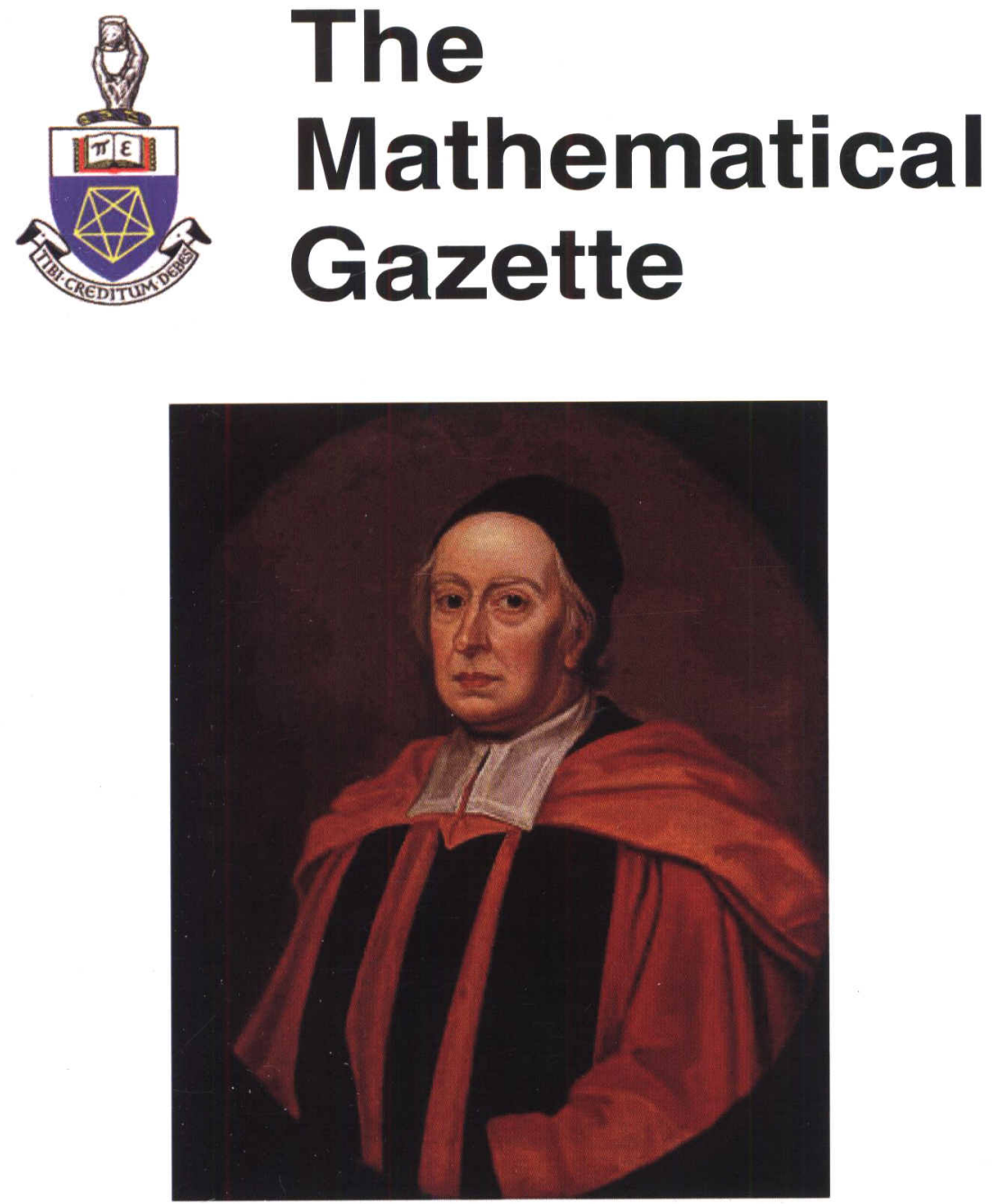

John Wallis and his product for $\pi$

\title{
Volume 94
}

Number 531

November 2010

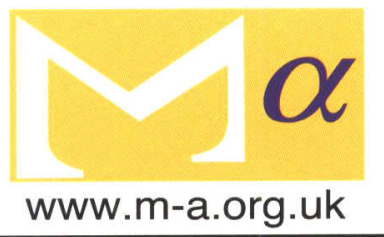




\section{THE MATHEMATICAL ASSOCIATION}

AN ASSOCIATION OF TEACHERS AND STUDENTS

OF ELEMENTARY MATHEMATICS

'I hold every man a debtor to his profession, from the which as men of course do seek to receive countenance and profit, so ought they of duty to endeavour themselves by way of amends to be a help and an ornament thereunto.'

BACON

\section{THE COUNCIL}

\section{PRESIDENT}

Dr David Acheson

\begin{tabular}{|c|c|c|c|}
\hline \multicolumn{3}{|c|}{ IMMEDIATE PAST PRESIDENT } & Mrs Jane Imrie \\
\hline \multicolumn{3}{|c|}{ PRESIDENT DESIGNATE } & Dr Paul Andrews \\
\hline \multicolumn{3}{|c|}{ CHAIR OF COUNCIL } & Professor Adam McBride \\
\hline \multicolumn{3}{|l|}{ SECRETARY } & Dr Catherine Ogden \\
\hline \multicolumn{3}{|l|}{ TREASURER } & Dr Paul Harris \\
\hline \multirow{2}{*}{$\begin{array}{l}\text { REPRESENTATIVES } \\
\text { OF COMMITTEES }\end{array}$} & \multicolumn{2}{|c|}{ BRANCHES } & Mr Keith Cadman \\
\hline & $\begin{array}{l}\text { CON } \\
\text { PROF } \\
\text { PUBI } \\
\text { PUBI } \\
\text { TEAC }\end{array}$ & $\begin{array}{l}\text { FERENCES } \\
\text { FESSIONAL DEVELOPMENT } \\
\text { LICATIONS } \\
\text { LICITY \& MEMBERSHIP } \\
\text { CHING COMMITTEE }\end{array}$ & $\begin{array}{l}\text { Mr Bill Richardson } \\
\text { Mrs Alison Clark-Wilson }(\mathrm{Ch} \\
\text { Mr Michael Fox } \\
\text { Dr Geoff Tennant } \\
\text { Dr Chris Pritchard }\end{array}$ \\
\hline \multicolumn{3}{|l|}{ EDITOR IN CHIEF } & \\
\hline \multicolumn{2}{|l|}{ OFFICE } & $\begin{array}{l}\text { Mrs Fiona Allan } \\
\text { Mr Mundher Adhami } \\
\text { Mr David Crawford } \\
\text { Mr Rob Eastaway }\end{array}$ & $\begin{array}{l}\text { Dr Tony Gardiner } \\
\text { Miss Mary Ledwick } \\
\text { Mrs Lynne McClure } \\
\text { Ms Rachael Read }\end{array}$ \\
\hline \multicolumn{3}{|c|}{ SENIOR ADMINISTRATOR } & \\
\hline \multicolumn{4}{|c|}{ EDITORIAL COMMITTEE OF THE MATHEMATICAL GAZETTE } \\
\hline \multicolumn{2}{|c|}{ Production Editor } & Mr Bill Richardson & \\
\hline \multicolumn{2}{|c|}{ Reviews Editor } & Dr Martin Griffiths & \\
\hline \multicolumn{2}{|c|}{ Problems Editors } & Mr Nick Lord, Dr Stan D & olan \\
\hline \multicolumn{2}{|c|}{ Assistant Editors } & $\begin{array}{l}\text { Mr Tom Roper, Mr Colin } \\
\text { Dr Andrew Jobbings, Pro }\end{array}$ & $\begin{array}{l}\text { Davis, Mr Michael Fox, } \\
\text { fessor Adam McBride }\end{array}$ \\
\hline
\end{tabular}

